\title{
Nonlocal Navier-Stokes problem with a small parameter
}

Veli B Shakhmurov

"Correspondence:
veli.sahmurov@okan.edu.tr
Department of Mechanical
Engineering, Okan University,
Akfirat, Tuzla, Istanbul, Turkey
Institute of Mathematics and
Mechanics, Azerbaijan National
Akademy of Science, Baku,
Azerbaijan

${ }^{*}$ Correspondence:

Akfirat, Tuzla, Istanbul, Turkey stitute of Mathematics and Akademy of Science, Baku,

\begin{abstract}
Initial nonlocal boundary value problems for a Navier-Stokes equation with a small parameter is considered. The uniform maximal regularity properties of the corresponding stationary Stokes operator, well-posedness of a nonstationary Stokes problem and the existence, uniqueness and uniformly $L^{p}$ estimates for the solution of the Navier-Stokes problem are established.
\end{abstract}

MSC: 35Q30; 76D05; 34G10; 35J25

Keywords: Stokes operators; Navier-Stokes equations; differential equations with small parameters; semigroups of operators; boundary value problems;

differential-operator equations; maximal $L_{p}$ regularity

\section{Introduction}

Consider the following Navier-Stokes problem with a parameter:

$$
\begin{gathered}
\frac{\partial u}{\partial t}-\triangle_{\varepsilon} u+(u \cdot \nabla) u+\nabla \varphi=f(x, t), \quad \operatorname{div} u=0, x \in G, t \in(0, T), \\
L_{k j \varepsilon} u=\sum_{i=0}^{m_{k j}} \varepsilon^{\sigma_{i}}\left[\alpha_{k j i} \frac{\partial^{i} u}{\partial x_{k}^{i}}\left(G_{k 0}\right)+\beta_{k j i} \frac{\partial^{i} u}{\partial x_{k}^{i}}\left(G_{k b}\right)\right]=0, \\
\quad k=1,2, \ldots, n, j=1,2, u(x, 0)=a(x),
\end{gathered}
$$

where

$$
\begin{aligned}
& \triangle_{\varepsilon} u=\varepsilon \sum_{k=1}^{n} \frac{\partial^{2} u}{\partial x_{k}^{2}}, \quad \sigma_{i}=\frac{1}{2}\left(i+\frac{1}{q}\right), \quad q \in(1, \infty), \\
& G=\prod_{k=1}^{n}\left(0, b_{k}\right), \quad G_{k 0}=\left(x_{1}, x_{2}, \ldots, x_{k-1}, 0, x_{k+1}, \ldots, x_{n}\right), \\
& G_{k b}=\left(x_{1}, x_{2}, \ldots, x_{k-1}, b_{k}, x_{k+1}, \ldots, x_{n}\right), \quad 0 \leq m_{k j} \leq 1,
\end{aligned}
$$

$\alpha_{k j i}, \beta_{k j i}$ are complex numbers, $\varepsilon$ is a small positive parameter,

$$
u=\left(u_{1}(x, t), u_{2}(x, t), \ldots, u_{n}(x, t)\right), \quad \varphi=\varphi(x, t)
$$

(c) 2013 Shakhmurov; licensee Springer. This is an Open Access article distributed under the terms of the Creative Commons Attribution License (http://creativecommons.org/licenses/by/2.0), which permits unrestricted use, distribution, and reproduction in any medium, provided the original work is properly cited. 
represent the unknown velocity and pressure, respectively,

$$
f=\left(f_{1}(x, t), f_{2}(x, t), \ldots, f_{n}(x, t)\right)
$$

represents a given external force and $a$ denotes the initial velocity. This problem is characterized by nonlocality of boundary conditions and by presence of a small term $\varepsilon$ which corresponds to the inverse of Reynolds number Re very large for the Navier-Stokes equations. From both the theoretical and computational points of view, singularly perturbed problems and asymptotic behavior of the Navier-Stokes equations with small viscosity when the boundary is either characteristic or non-characteristic have been well studied; see, e.g., [1-6]. In the present work, we established a uniform time of existence and estimates for solutions of problem (1.1)-(1.3). It is clear that for $\varepsilon=1$, choosing the boundary conditions locally and $m_{k j}=0$, problem (1.1)-(1.3) is reduced to the classical Navier-Stokes problem

$$
\begin{aligned}
& \frac{\partial u}{\partial t}-\Delta u+(u \cdot \nabla) u+\nabla \varphi=f(x, t), \quad \operatorname{div} u=0, x \in \Omega, t \in(0, T), \\
& \left.u\right|_{\partial \Omega}=0, \quad u(x, 0)=a(x) .
\end{aligned}
$$

Note that the existence of weak or strong solutions and regularity properties of classical Navier-Stokes problems were extensively studied, e.g., in [1-3, 5, 7-33]. There is extensive literature on the solvability of the initial value problem for the Navier-Stokes equation ( see, e.g., [25] for further papers cited there ). Hopf [20] proved the existence of a global weak solution of (1.4) using the Faedo-Galerkin approximation and an energy inequality. Another approach to problem (1.4) is to use semigroup theory. Kato and Fujita [18, 22, 34] and Sobolevskii [27] transformed equation (1.4) into an evolution equation in the Hilbert space $L^{2}$. They proved the existence of a unique global strong solution for any squaresummable initial velocity when $n=2$. On the other hand, when $n=3$ they proved the existence of a unique local strong solution if the initial velocity has some regularity. Other contributions in this field have also assumed some regularity of the initial velocity corresponding to the Stokes problem; see, for example, Solonnikov [26] and Heywood [21]. Afterward, Giga and Sohr [13] improved this result in two directions. First, they generalized the result of Solonnikov for spaces with different exponents in space and time, and the estimate obtained was global in time. Here, first at all, we consider the nonlocal (boundary value problem) BVP for the following differential operator equation (DOE) with small parameters:

$$
\begin{aligned}
& -\triangle_{\varepsilon} u+(A+\lambda) u=f(x), \quad x \in G, \\
& L_{k j \varepsilon} u=\sum_{i=0}^{m_{k j}} \varepsilon_{k}^{\sigma_{i}}\left[\alpha_{k j i} \frac{\partial^{i} u}{\partial x_{k}^{i}}\left(G_{k 0}\right)+\beta_{k j i} \frac{\partial^{i} u}{\partial x_{k}^{i}}\left(G_{k b}\right)\right]=0, \\
& \quad k=1,2, \ldots, n, j=1,2,
\end{aligned}
$$

where $A$ is a linear operator in a Banach space $E, \alpha_{k j i}, \beta_{k j i}$ are complex numbers, $\varepsilon_{k}$ are positive and $\lambda$ is a complex parameter. We show that problem (1.5) has a unique solution $u \in W^{2, q}(G ; E(A), E)$ for $f \in W^{m, q}(G ; E)$ and $\lambda \in S_{\psi, \varkappa}$ with sufficiently large $\varkappa>0$, and the 
following coercive uniform estimate holds:

$$
\sum_{k=1}^{n} \sum_{i=0}^{m+2} \varepsilon_{k}^{\frac{i}{m+2}}|\lambda|^{1-\frac{i}{2}}\left\|\frac{\partial^{i} u}{\partial x_{k}^{i}}\right\|_{L^{q}(G ; E)}+\|A u\|_{L^{q}(G ; E)} \leq C\|f\|_{W^{m, q}(G ; E)}, \quad m \geq 0
$$

with $C(q)$ independent of $\varepsilon_{1}, \varepsilon_{2}, \ldots, \varepsilon_{n}, \lambda$ and $f$.

Further, we consider the nonlocal BVP for the stationary Stokes system with small parameters

$$
\begin{gathered}
\triangle_{\varepsilon} u+\nabla \varphi=f(x, t), \quad \operatorname{div} u=0, x \in G, t \in(0, T), \\
L_{k j \varepsilon} u=\sum_{i=0}^{m_{k j}} \varepsilon_{k}^{\sigma_{i}}\left[\alpha_{k j i} \frac{\partial^{i} u}{\partial x_{k}^{i}}\left(G_{k 0}\right)+\beta_{k j i} \frac{\partial^{i} u}{\partial x_{k}^{i}}\left(G_{k b}\right)\right]=0, \\
k=1,2, \ldots, n, j=1,2, u(x, 0)=0,
\end{gathered}
$$

where

$$
u=\left(u_{1}(x, t), u_{2}(x, t), \ldots, u_{n}(x, t)\right), \quad f=\left(f_{1}(x, t), f_{2}(x, t), \ldots, f_{n}(x, t)\right)
$$

Then we consider the initial nonlocal BVP for the following nonstationary Stokes equation with small parameters:

$$
\begin{gathered}
\frac{\partial u}{\partial t}-\triangle_{\varepsilon} u+\nabla \varphi=f(x, t), \quad \operatorname{div} u=0, x \in G, t \in(0, T), \\
L_{k j \varepsilon} u=\sum_{i=0}^{m_{k j}} \varepsilon_{k}^{\sigma_{i}}\left[\alpha_{k j i} \frac{\partial^{i} u}{\partial x_{k}^{i}}\left(G_{k 0}\right)+\beta_{k j i} \frac{\partial^{i} u}{\partial x_{k}^{i}}\left(G_{k b}\right)\right]=0, \\
k=1,2, \ldots, n, j=1,2, u(x, 0)=0 .
\end{gathered}
$$

Problem (1.7) can be expressed as the abstract parabolic problem with a parameter

$$
\frac{d u}{d t}+O_{\varepsilon, q} u=f(t), \quad u(0)=0
$$

where $O_{\varepsilon, q}$ is a stationary parameter depending on the Stokes operator in a solenoidal space $L_{\sigma}^{q}\left(G ; R^{n}\right)$ defined by

$$
\begin{aligned}
& D\left(O_{\varepsilon, q}\right)=W_{\sigma}^{2, q}\left(G, L_{k j \varepsilon}\right)=\left\{u \in W^{2, q}(G), \operatorname{div} u=0, L_{k j \varepsilon} u=0, k=1,2, \ldots, n, j=1,2\right\}, \\
& O_{\varepsilon, q} u=-P \triangle_{\varepsilon} u .
\end{aligned}
$$

We prove that the operator $O_{\varepsilon, q}$ is positive in $L^{q}\left(G ; R^{n}\right)$ uniformly with respect to parameters $\varepsilon=\left(\varepsilon_{1}, \varepsilon_{2}, \ldots, \varepsilon_{n}\right)$ and also is a generator of a holomorphic semigroup. Then, by using $L^{p}$-maximal regularity theorems (see, e.g., $\left.[33,35]\right)$ for abstract parabolic equations (1.8), we obtain that for every $f \in L^{p}\left(0, T ; L^{q}\left(\Omega ; R^{n}\right)\right)=B(p, q), p, q \in(1, \infty)$, there is a unique solution $(u, \nabla \varphi)$ of problem (1.8) and the following uniform estimate holds:

$$
\left\|\frac{\partial u}{\partial t}\right\|_{B(p, q)}+\sum_{k=1}^{n}\left\|\varepsilon_{k} \frac{\partial^{2} u}{\partial x_{k}^{2}}\right\|_{B(p, q)}+\|\nabla \varphi\|_{B(p, q)} \leq C\|f\|_{B(p, q)}
$$


with $C=C(T, \Omega, p, q)$ independent of $f$ and $\varepsilon$. Afterwords, by using the above uniform coercive estimate, we derive local uniform existence and uniform a priori estimates of a solution of problem (1.1)-(1.3).

Modern analysis methods, particularly abstract harmonic analysis, the operator theory, the interpolation of Banach spaces, the theory of semigroups of linear operators, embedding and trace theorems in vector-valued Sobolev-Lions spaces are the main tools implemented to carry out the analysis.

\section{Notations, definitions and background}

Let $E$ be a Banach space and $L^{p}(\Omega ; E)$ denotes the space of strongly measurable $E$-valued functions that are defined on the measurable subset $\Omega \subset R^{n}$ with the norm

$$
\|f\|_{L^{p}}=\|f\|_{L^{p}(\Omega ; E)}=\left(\int_{\Omega}\|f(x)\|_{E}^{p} d x\right)^{\frac{1}{p}}, \quad 1 \leq p<\infty
$$

The Banach space $E$ is called a UMD-space if the Hilbert operator

$$
(H f)(x)=\lim _{\varepsilon \rightarrow 0} \int_{|x-y|>\varepsilon} \frac{f(y)}{x-y} d y
$$

is bounded in $L_{p}(R, E), p \in(1, \infty)$ (see, e.g., [36]). UMD spaces include, e.g., $L_{p}, l_{p}$ spaces and Lorentz spaces $L_{p q}, p, q \in(1, \infty)$.

Let $\mathbb{C}$ be the set of complex numbers and

$$
\begin{aligned}
& S_{\psi}=\{\lambda \in \mathbb{C},|\arg \lambda| \leq \varphi \cup\{0\}, 0 \leq \psi<\pi\}, \\
& S_{\psi, \varkappa}=\left\{\lambda \in S_{\psi},|\lambda|>\varkappa>0\right\} .
\end{aligned}
$$

A linear operator $A$ is said to be $\psi$-positive in a Banach space $E$ with bound $M>0$ if $D(A)$ is dense on $E$ and $\left\|(A+\lambda I)^{-1}\right\|_{B(E)} \leq M(1+|\lambda|)^{-1}$ for any $\lambda \in S_{\psi}, 0 \leq \psi<\pi$, where $I$ is the identity operator in $E, B(E)$ is the space of bounded linear operators in $E$. It is known $[30, \S 1.15 .1]$ that there exist the fractional powers $A^{\theta}$ of a positive operator $A$. Let $E\left(A^{\theta}\right)$ denote the space $D\left(A^{\theta}\right)$ with the norm

$$
\|u\|_{E\left(A^{\theta}\right)}=\left(\|u\|^{p}+\left\|A^{\theta} u\right\|^{p}\right)^{\frac{1}{p}}, \quad 1 \leq p<\infty, 0<\theta<\infty .
$$

Let $\mathbb{N}$ denote the set of natural numbers. A set $G \subset B\left(E_{1}, E_{2}\right)$ is called $R$-bounded (see, e.g., [36]) if there is a positive constant $C$ such that for all $T_{1}, T_{2}, \ldots, T_{m} \in G$ and $u_{1}, u_{2}, \ldots, u_{m} \in E_{1}, m \in \mathbb{N}$,

$$
\int_{\Omega}\left\|\sum_{j=1}^{m} r_{j}(y) T_{j} u_{j}\right\|_{E_{2}} d y \leq C \int_{\Omega}\left\|\sum_{j=1}^{m} r_{j}(y) u_{j}\right\|_{E_{1}} d y,
$$

where $\left\{r_{j}\right\}$ is a sequence of independent symmetric $\{-1,1\}$-valued random variables on $\Omega$. The smallest $C$ for which the above estimate holds is called an $R$-bound of the collection $G$ and denoted by $R(G)$. 
A set $G_{h} \subset B\left(E_{1}, E_{2}\right)$ is called uniform $R$-bounded if there is a constant $C$ independent of $h \in Q$ such that for all $T_{1}(h), T_{2}(h), \ldots, T_{m}(h) \in G_{h}$ and $u_{1}, u_{2}, \ldots, u_{m} \in E_{1}, m \in \mathbb{N}$,

$$
\int_{\Omega}\left\|\sum_{j=1}^{m} r_{j}(y) T_{j}(h) u_{j}\right\|_{E_{2}} d y \leq C \int_{\Omega}\left\|\sum_{j=1}^{m} r_{j}(y) u_{j}\right\|_{E_{1}} d y,
$$

which implies that $\sup _{h \in Q} R\left(G_{h}\right) \leq C$.

The $\psi$-positive operator $A$ is said to be $R$-positive in a Banach space $E$ if the set $L_{A}=$ $\left\{\xi(A+\xi)^{-1}: \xi \in S_{\psi}\right\}, 0 \leq \psi<\pi$, is $R$-bounded.

The operator $A(t)$ is said to be $\psi$-positive in $E$ uniformly in $t \in \sigma$ with bound $M>0$ if $D(A(t))$ is independent of $t, D(A(t))$ is dense in $E$ and $\left\|(A(t)+\lambda)^{-1}\right\| \leq M(1+|\lambda|)^{-1}$ for all $\lambda \in S_{\psi}, 0 \leq \psi<\pi$, where $M$ does not depend on $t$ and $\lambda$.

Let $E_{0}$ and $E$ be two Banach spaces, and let $E_{0}$ be continuously and densely embedded into $E$. Let $\Omega$ be a measurable set in $R^{n}$ and $m$ be a positive integer. Let $W^{m, p}\left(\Omega ; E_{0}, E\right)$ denote the space consisting of all functions $u \in L^{p}\left(\Omega ; E_{0}\right)$ that have the generalized derivatives $\frac{\partial^{m} u}{\partial x_{k}^{m}} \in L^{p}(\Omega ; E)$, with the norm

$$
\|u\|_{W^{m, p}\left(\Omega ; E_{0}, E\right)}=\|u\|_{L^{p}\left(\Omega ; E_{0}\right)}+\sum_{k=1}^{n}\left\|\frac{\partial^{m} u}{\partial x_{k}^{m}}\right\|_{L^{p}(\Omega ; E)}<\infty
$$

For $n=1, \Omega=(a, b), a, b \in \mathbb{N}$, the space $W^{m, p}\left(\Omega ; E_{0}, E\right)$ will be denoted by $W^{m, p}(a, b$; $\left.E_{0}, E\right)$.

Sometimes we use one and the same symbol $C$ without distinction in order to denote positive constants which may differ from each other even in a single context. When we want to specify the dependence of such a constant on a parameter, say $\alpha$, we write $C_{\alpha}$.

\section{Boundary value problems for abstract elliptic equations}

In this section, we consider problem (1.5). We derive the maximal regularity properties of this problem.

It should be noted that BVPs for DOEs were studied, e.g., in [35-38] and [6, 26, 27, 39-43]. For references, see [35, 43]. Let $\alpha_{k j}=\alpha_{k j m_{k}}$ and $\beta_{k j}=\beta_{k j m_{k}}$. First, we prove the following theorem.

Theorem 3.1 Let the following conditions be satisfied:

(1) $E$ is a UMD space and $A$ is an R-positive operator in $E$ for $0 \leq \psi<\pi$;

(2) $q \in(1, \infty), \eta_{k}=(-1)^{m_{1}} \alpha_{k 1} \beta_{k 2}-(-1)^{m_{2}} \alpha_{k 2} \beta_{k 1} \neq 0,0<t_{k} \leq 1, k=1,2, \ldots, n$.

Then problem (1.5) has a unique solution $u \in W^{2, q}(G ; E(A), E)$ for $f \in L^{q}(G ; E)$ and $\lambda \in$ $S_{\psi, \varkappa}$ with sufficiently large $\varkappa>0$. Moreover, the following coercive uniform estimate holds:

$$
\sum_{k=1}^{n} \sum_{j=0}^{2} \varepsilon_{k}^{\frac{j}{2}}|\lambda|^{1-\frac{j}{2}}\left\|\frac{\partial^{j} u}{\partial x_{k}^{j}}\right\|_{L^{q(G ; E)}}+\|A u\|_{L^{q}(G ; E)} \leq C\|f\|_{L^{q}(G ; E)}
$$

with $C(q)$ independent of $\varepsilon_{1}, \varepsilon_{2}, \ldots, \varepsilon_{n}, \lambda$ and $f$.

Proof Let us consider the BVP

$$
-\varepsilon_{1} \frac{\partial^{2} u}{\partial x_{1}^{2}}-\varepsilon_{2} \frac{\partial^{2} u}{\partial x_{2}^{2}}+(A+\lambda) u\left(x_{1}, x_{2}\right)=f\left(x_{1}, x_{2}\right), \quad L_{k j \varepsilon} u=0, \quad k, j=1,2,
$$


where $x_{1}, x_{2} \in G_{2}=\left(0, b_{1}\right) \times\left(0, b_{2}\right), L_{k j \varepsilon}$ are defined by equalities (3.1)-(3.2). For the investigation (3.4), we consider the following BVP for ordinary DOE:

$$
L u=-\varepsilon_{2} \frac{d^{2} u}{d x_{2}^{2}}+(A+\lambda) u\left(x_{2}\right)=f\left(x_{2}\right), \quad y \in\left(0, b_{2}\right), \quad L_{2 j \varepsilon_{2}} u=0
$$

where $L_{2 k}$ are boundary conditions of type (1.5) on $\left(0, b_{2}\right)$. By virtue of [40, Theorem 3.2], we obtain that problem (3.3) has a unique solution $u \in W^{2, q}\left(0, b_{2} ; E(A), E\right)$ for $f \in L^{q}\left(0, b_{2} ; E\right), \lambda \in S_{\psi, \varkappa}$, with sufficiently large $\varkappa>0$, and the following coercive uniform estimate holds:

$$
\sum_{j=0}^{2} \varepsilon_{2}^{\frac{j}{2}}|\lambda|^{1-\frac{j}{2}}\left\|u^{(j)}\right\|_{L^{q}\left(0, b_{2} ; E\right)}+\|A u\|_{L^{q}\left(0, b_{2} ; E\right)} \leq C\|f\|_{L^{q}\left(0, b_{2} ; E\right)} .
$$

Since $L^{q}\left(0, b_{1} ; L^{q}\left(0, b_{2} ; E\right)\right)=L^{q}\left(G_{2} ; E\right)$, problem (3.2) can be expressed as the following problem:

$$
-\varepsilon_{1} \frac{d u}{d x_{1}^{2}}+\left(B_{\varepsilon_{2}}+\lambda\right) u\left(x_{1}\right)=f\left(x_{1}\right), \quad L_{k 1 \varepsilon_{1}} u=0
$$

where $B_{\varepsilon_{2}}$ is the differential operator in $L^{q}\left(0, b_{1} ; E\right)$ generated by problem (3.3), i.e.,

$$
D\left(B_{\varepsilon_{2}}\right)=W^{2, q}\left(0, b_{2} ; E(A), E, L_{2 k \varepsilon_{2}}\right), \quad B_{\varepsilon_{2}} u=-\varepsilon_{2} \frac{d^{2} u}{d x_{2}^{2}}+A u\left(x_{2}\right) .
$$

By virtue of [35, Theorem 4.5.2], $F=L^{q}\left(0, b_{2} ; E\right) \in \mathrm{UMD}$ provided $E \in \mathrm{UMD}, q \in(1, \infty)$. Hence, by virtue of [40, Theorem 3.2] and [41, Theorem 3.1], the operator $B_{\varepsilon_{2}}$ is uniformly $R$-positive in $F$. Then, by applying again [40, Theorem 3.2], we get that for $f \in$ $L^{q}\left(0, b_{1} ; F\right), \lambda \in S_{\psi, \varkappa}$ and sufficiently large $\varkappa>0$, problem (3.5) has a unique solution $u \in$ $W^{2, q}\left(0, b_{1} ; D(B), F\right)$, and the following coercive uniform estimate holds:

$$
\sum_{j=0}^{2} \varepsilon_{1}^{\frac{j}{2}}|\lambda|^{1-\frac{j}{2}}\left\|u^{(j)}\right\|_{L^{q\left(0, b_{1} ; F\right)}}+\left\|B_{\varepsilon_{2}} u\right\|_{L^{q}\left(0, b_{1} ; F\right)} \leq C\|f\|_{L^{q}\left(0, b_{1} ; F\right)} .
$$

The estimate (3.4) implies the uniform estimate

$$
\sum_{j=0}^{2} \varepsilon_{2}^{\frac{j}{2}}|\lambda|^{1-\frac{j}{2}}\left\|u^{(j)}\right\|_{L^{q\left(0, b_{1} ; F\right)}} \leq C\left\|B\left(\varepsilon_{2}\right) u\right\|_{L^{q}\left(0, b_{1} ; F\right)} .
$$

By using (3.4) and (3.7), we obtain that problem (3.7) has a unique solution $u \in W^{2, q}\left(G_{2}\right.$; $E(A), E)$ for $f \in L^{q}\left(G_{2} ; E\right), \lambda \in S_{\psi, \varkappa}$ with sufficiently large $\varkappa>0$, and the coercive uniform estimate holds

$$
\sum_{k=1}^{2} \sum_{j=0}^{2} \varepsilon_{k}^{\frac{j}{2}}|\lambda|^{1-\frac{j}{2}}\left\|\frac{\partial^{j} u}{\partial x_{k}^{j}}\right\|_{L^{q}\left(G_{2} ; E\right)}+\|A u\|_{L^{q}\left(G_{2} ; E\right)} \leq C\|f\|_{L^{q}\left(G_{2} ; E\right)} .
$$

Further, by continuing this process $n$-times, we obtain the assertion.

From Theorem 3.1 we obtain the following. 
Corollary 3.1 Let $0<\varepsilon_{k} \leq 1,(-1)^{m_{k 1}} \alpha_{k 1} \beta_{k 2}-(-1)^{m_{k 2}} \alpha_{k 2} \beta_{k 1} \neq 0$. For $f \in L^{q}\left(G ; R^{n}\right), q \in$ $(1, \infty)$ and for $\lambda \in S_{\psi, \varkappa}$ with sufficiently large $\varkappa>0$, there is a unique solution $u$ of problem (1.5) and the following uniform coercive estimate holds:

$$
\sum_{k=1}^{n} \sum_{i=0}^{2}|\lambda|^{1-\frac{i}{2}} \varepsilon_{k}^{\frac{i}{2}}\left\|\frac{\partial^{i} u}{\partial x_{k}^{i}}\right\|_{q} \leq C\|f\|_{q}
$$

with $C=C(q)$ independent of $f, \varepsilon_{k}$ and $\lambda$.

Proof Let us put $E=R^{n}$ and $A=\varkappa>0$ in Theorem 3.1. It is known that the operator $A=$ $\varkappa>0$ is $R$-positive in $R^{n}$ (see, e.g., [36]). So, the estimate (3.1) implies Corollary 3.1.

Consider the differential operator $Q_{\varepsilon}$ generated by problem (1.5), i.e.,

$$
\begin{aligned}
& D\left(Q_{\varepsilon}\right)=W^{2, q}\left(G ; L_{k j \varepsilon}\right)=\left\{u \in W^{2, q}(G), L_{k j \varepsilon} u=0, k=1,2, \ldots, n, j=1,2\right\}, \\
& Q_{\varepsilon} u=-\triangle_{\varepsilon} u+A u .
\end{aligned}
$$

From Theorem 3.1 we obtain the following.

Result 3.1 For $\lambda \in S_{\psi, \varkappa}$, there is a resolvent $\left(Q_{\varepsilon}+\lambda\right)^{-1}$ of the operator $Q_{\varepsilon}$ satisfying the following uniform estimate:

$$
\sum_{k=1}^{n} \sum_{i=0}^{2}|\lambda|^{1-\frac{i}{2}} \varepsilon_{k}^{\frac{i}{2}}\left\|\frac{\partial^{i}}{\partial x_{k}^{i}}\left(Q_{\varepsilon}+\lambda\right)^{-1}\right\|_{B\left(L^{q}(G ; E)\right)} \leq C
$$

It is clear that the solution $u$ of problem (1.5) depends on parameters $\varepsilon=\left(\varepsilon_{1}, \varepsilon_{2}, \ldots, \varepsilon_{n}\right)$, i.e., $u=u(x, \varepsilon)$. In view of Theorem 3.1, we established estimates for the solution of (1.5) uniformly in $\varepsilon_{1}, \varepsilon_{2}, \ldots, \varepsilon_{n}$.

\section{Regularity properties of solutions for DOEs with parameters}

In this section, we show the separability properties of problem (1.5) in Sobolev spaces $W^{m, q}(G ; E)$. The main result is the following theorem.

Theorem 4.1 Let the following conditions be satisfied:

(1) $E$ is a UMD space and $A$ is an R-positive operator in $E$;

(2) $m$ is a positive integer $q \in(1, \infty), 0<t_{k} \leq 1$, and

$$
\eta_{k}=(-1)^{m_{1}} \alpha_{k 1} \beta_{k 2}-(-1)^{m_{2}} \alpha_{k 2} \beta_{k 1} \neq 0, \quad k=1,2, \ldots, n .
$$

Then problem (3.1)-(3.2) has a unique solution $u \in W^{2+m, q}(G ; E(A), E)$ for $f \in W^{m, q}(G$; $E), \lambda \in S_{\psi, \varkappa}$, with sufficiently large $\varkappa>0$, and the following coercive uniform estimate holds:

$$
\sum_{k=1}^{n} \sum_{i=0}^{m+2} \varepsilon_{k}^{\frac{i}{m+2}}|\lambda|^{1-\frac{i}{m+2}}\left\|\frac{\partial^{i} u}{\partial x_{k}^{i}}\right\|_{W^{m, q(G ; E)}}+\|A u\|_{L^{q}(G ; E)} \leq C\|f\|_{W^{m, q(G ; E)}}
$$

with $C=C(q, A)$ independent of $\varepsilon_{1}, \varepsilon_{2}, \ldots, \varepsilon_{n}, \lambda$ and $f$. 
Consider first the following nonlocal BVP for an ordinary DOE with a small parameter:

$$
\begin{aligned}
& \left(L_{t}+\lambda\right) u=-t u^{(2)}(x)+(A+\lambda) u(x)=f(x), \quad x \in(0,1), \\
& L_{k t} u=\sum_{i=0}^{m_{k}} t^{\sigma_{i}}\left[\alpha_{k i} u^{(i)}(0)+\beta_{k i} u^{(i)}(1)\right]=f_{k}, \quad k=1,2,
\end{aligned}
$$

where $\sigma_{i}=\frac{i}{2}+\frac{1}{2 p}, m_{k} \in\{0, m+1\}, \alpha_{k i}, \beta_{k i}$ are complex numbers, $t$ is positive, $\lambda$ is a complex parameter and $A$ is a linear operator in $E$. Let $A_{\lambda}=A+\lambda I$.

To prove the main result, we need the following result in [37, Theorem 2.1].

Theorem A Let $E$ be a UMD space, $A$ be a $\psi$-positive operator in $E$ with bound $M, 0 \leq$ $\psi<\pi$. Let $m$ be a positive integer, $1<p<\infty$, and $\alpha \in\left(\frac{1}{2 p}, \frac{1}{2 p}+m\right)$. Then, for $\lambda \in S_{\varphi}$, an operator $-A_{\lambda}^{\frac{1}{2}}$ generates a semigroup $e^{-x A_{\lambda}^{\frac{1}{2}}}$ which is holomorphic for $x>0$. Moreover, there exists a positive constant $C$ (depending only on $M, \psi, m, \alpha$ and $p$ ) such that for every $u \in$ $\left(E, E\left(A^{m}\right)\right)_{\frac{\alpha}{m}-\frac{1}{2 m p}, p}$ and $\lambda \in S_{\psi}$,

$$
\int_{0}^{\infty}\left\|A_{\lambda}^{\alpha} e^{-x A_{\lambda}^{\frac{1}{2}}} u\right\|^{p} d x \leq C\left[\|u\|_{\left(E, E\left(A^{m}\right)\right)_{\frac{\alpha}{m}-\frac{1}{2 m p}, p}^{p}}^{p}+|\lambda|^{\alpha p-\frac{1}{2}}\|u\|_{E}^{p}\right] .
$$

In a similar way as in $[43, \S 1.8 .2$, Theorem 2$]$, we obtain the following lemma.

Lemma 4.1 Let $m$ and $j$ be integer numbers, $0 \leq j \leq m-1, \theta_{j}=\frac{p j+1}{p m}, 0<t \leq 1, x_{0} \in[0, b]$. Then, for $u \in W_{p}^{m}\left(0, b ; E_{0}, E\right)$, the transformation $u \rightarrow u^{(j)}\left(x_{0}\right)$ is bounded linear from $W_{p}^{m}\left(0, b ; E_{0}, E\right)$ onto $\left(E_{0}, E\right)_{\theta_{j}, p}$ and the following inequality holds:

$$
t^{\theta_{j}}\left\|u^{(j)}\left(x_{0}\right)\right\|_{\left(E_{0}, E\right)_{\theta_{j}, p}} \leq C\left(\left\|t u^{(m)}\right\|_{L_{p}(0, b ; E)}+\|u\|_{L_{p}\left(0, b ; E_{0}\right)}\right) .
$$

Consider at first the homogeneous problem of (4.2)

$$
\begin{aligned}
& \left(L_{t}+\lambda\right) u=-t u^{(2)}(x)+(A+\lambda) u(x)=0, \\
& L_{k t} u=\sum_{i=0}^{m_{k}} t^{\sigma_{i}}\left[\alpha_{k i} u^{(i)}(0)+\beta_{k i} u^{(i)}(1)\right]=f_{k}, \quad k=1,2 .
\end{aligned}
$$

Let

$$
E_{k}=(E(A), E)_{\theta_{k}, p}
$$

Lemma 4.2 Let $A$ be an R-positive operator in a UMD space $E$ and

$$
0<t \leq 1, \quad \eta=(-1)^{m_{1}} \alpha_{1} \beta_{2}-(-1)^{m_{2}} \alpha_{2} \beta_{1} \neq 0 .
$$

Then problem (4.3) has a unique solution $u \in W^{m+2, p}(0,1 ; E(A), E)$ for $f_{k} \in E_{k}, p \in(1, \infty)$, $\lambda \in S_{\psi}$, and the coercive uniform estimate holds

$$
\sum_{i=0}^{m+2} t^{\frac{i}{m+2}}|\lambda|^{1-\frac{i}{m+2}}\left\|u^{(i)}\right\|_{L^{p}(0,1 ; E)}+\|A u\|_{L^{p}(0,1 ; E)} \leq M \sum_{k=1}^{2}\left(\left\|f_{k}\right\|_{E_{k}}+|\lambda|^{1-\theta_{k}}\left\|f_{k}\right\|_{E}\right) .
$$


Proof In a similar way as in [40, Theorem 3.1], we obtain the representation of the solution of (4.3)

$$
\begin{aligned}
u(x)= & t^{-\frac{1}{2 p}}\left\{e^{-x t^{-\frac{1}{2}} A_{\lambda}^{\frac{1}{2}}}\left[C_{11}+d_{11}(\lambda, t)\right]+e^{-(1-x) t^{-\frac{1}{2}} A_{\lambda}^{\frac{1}{2}}}\left[C_{12}+d_{12}(\lambda, t)\right]\right\} A_{\lambda}^{-\frac{m_{1}}{2}} f_{1} \\
& +t^{-\frac{1}{2 p}}\left\{e^{-x t^{-\frac{1}{2}} A_{\lambda}^{\frac{1}{2}}}\left[C_{21}+d_{21}(\lambda, t)\right]+e^{-(1-x) t^{-\frac{1}{2}}} A_{\lambda}^{\frac{1}{2}}\left[C_{22}+d_{22}(\lambda, t)\right]\right\} A_{\lambda}^{-\frac{m_{2}}{2}} f_{2},
\end{aligned}
$$

where $C_{i j}$ and $d_{i j}$ are uniformly bounded operators. Then, in view of positivity of $A$, we obtain from (4.5)

$$
\begin{aligned}
& \sum_{i=0}^{m+2} t^{\frac{i}{m+2}}|\lambda|^{1-\frac{i}{m+2}}\left\|u^{(i)}\right\|_{L^{p}(0,1 ; E)}+\|A u\|_{L^{p}(0,1 ; E)} \\
& \leq C t^{-\frac{1}{2 p}} \sum_{k=1}^{2}\left[\sum_{i=0}^{m+2}|\lambda|^{\mu_{i}}\left\|A_{\lambda}^{-\frac{\left(m_{k}-i\right)}{2}}\left[e^{-(1-x) t^{-\frac{1}{2}} A_{\lambda}^{\frac{1}{2}}}+e^{-x t^{-\frac{1}{2}}} A_{\lambda}^{\frac{1}{2}}\right] f_{k}\right\|_{L^{p}(0,1 ; E)}\right. \\
& \left.+\left\|A A_{\lambda}^{-\frac{m_{k}}{2}} e^{-x t^{-\frac{1}{2}} A_{\lambda}^{\frac{1}{2}}} f_{k}\right\|_{L^{p}(0,1 ; E)}\right] \leq C t^{-\frac{1}{2 p}}, \\
& \sum_{k=1}^{2}\left[\sum_{i=0}^{m+2}|\lambda|^{1-\frac{i}{2}}\left\|A_{\lambda}^{-\left(1-\frac{m m_{k}}{2(m+2)}+\frac{i}{2}\right)}\right\|\left\|A_{\lambda}^{1-\frac{m_{k}}{m+2}} e^{-x t^{-\frac{1}{2}} A_{\lambda}^{\frac{1}{2}}} f_{k}\right\|_{L^{p}(0,1 ; E)}\right. \\
& \left.+\left\|A A_{\lambda}^{-\frac{m_{k}}{m+2}}\left[e^{-x t^{-\frac{1}{2}}} A_{\lambda}^{\frac{1}{2}}+e^{-(1-x) t^{-\frac{1}{2}}} A_{\lambda}^{\frac{1}{2}}\right] f_{k}\right\|_{L^{p}(0,1 ; E)}\right] \\
& \leq C t^{-\frac{1}{2 p}} \sum_{k=1}^{2}\left[\left(\int_{0}^{1}\left\|A_{\lambda}^{1-\frac{m_{k}}{m+2}}\left[e^{-x t^{-\frac{1}{2}}} A_{\lambda}^{\frac{1}{2}}+e^{-(1-x) t^{-\frac{1}{2}} A_{\lambda}^{\frac{1}{2}}}\right] f_{k}\right\|^{p} d x\right)^{\frac{1}{p}}\right. \\
& \left.+\left(\int_{0}^{1}\left\|A A_{\lambda}^{-\frac{m_{k}}{m+2}}\left[e^{-x t^{-\frac{1}{2}} A_{\lambda}^{\frac{1}{2}}}+e^{-(1-x) t^{-\frac{1}{2}} A_{\lambda}^{\frac{1}{2}}}\right] f_{k}\right\|^{p} d x\right)^{\frac{1}{p}}\right] .
\end{aligned}
$$

By changing the variable $x t^{-\frac{1}{2}}=y$ and in view of Theorem A, we obtain

$$
\begin{aligned}
& t^{-\frac{1}{2 p}}\left(\int_{0}^{1}\left\|A_{\lambda}^{-\frac{m_{k}}{m+2}}\left[e^{-x t^{-\frac{1}{2}} A_{\lambda}^{\frac{1}{2}}}+e^{-(1-x) t^{-\frac{1}{2}} A_{\lambda}^{\frac{1}{2}}}\right] f_{k}\right\|^{p} d x\right)^{\frac{1}{p}} \\
& \quad \leq M_{1} \sum_{k=1}^{2}\left[\left\|f_{k}\right\|_{E_{k}}+|\lambda|^{1-\theta_{k}}\left\|f_{k}\right\|\right] .
\end{aligned}
$$

By using the estimate (4.8), by virtue of Theorem A, we get the uniform estimate

$$
\begin{aligned}
& \sum_{k=1}^{2} t^{-\frac{1}{2 p}}\left(\int_{0}^{1}\left\|A A_{\lambda}^{-\frac{m_{k}}{m+2}}\left[e^{-x t^{-\frac{1}{2}} A_{\lambda}^{\frac{1}{2}}}+e^{-(1-x) t^{-\frac{1}{2}} A_{\lambda}^{\frac{1}{2}}}\right] f_{k}\right\|^{p} d x\right)^{\frac{1}{p}} \\
& \quad \leq M_{2} \sum_{k=1}^{2}\left[\left\|f_{k}\right\|_{E_{k}}+|\lambda|^{1-\theta_{k}}\left\|f_{k}\right\|\right] .
\end{aligned}
$$

Then from (4.6)-(4.9) we obtain (4.4).

Now we can represent a more general result for nonhomogeneous problem (4.2). 
Theorem 4.2 Assume that the following conditions are satisfied:

(1) $E$ is a UMD space and $A$ is an R-positive operator in $E$;

(2) $\eta=(-1)^{m_{1}} \alpha_{1} \beta_{2}-(-1)^{m_{2}} \alpha_{2} \beta_{1} \neq 0, \theta_{k}=\frac{m_{k}}{m+2}+\frac{1}{2 p}, k=1,2,0<t \leq 1, p \in(1, \infty)$.

Then the operator $u \rightarrow\left\{\left(L_{t}+\lambda\right) u, L_{1 t} u, L_{2 t} u\right\}$ is an isomorphism from $W^{m+2, p}(0,1 ; E(A), E)$ onto $W^{m, p}(0,1 ; E) \times E_{1} \times E_{2}$ for $\lambda \in S_{\psi, \varkappa}$ with large enough $\varkappa>0$. Moreover, the uniform coercive estimate holds

$$
\begin{aligned}
& \sum_{j=0}^{m+2} t^{\frac{j}{m+2}}|\lambda|^{1-\frac{j}{m+2}}\left\|u^{(j)}\right\|_{L^{p}(0,1 ; E)}+\|A u\|_{L^{p}(0,1 ; E)} \\
& \quad \leq C\left[\|f\|_{W^{m, p}(0,1 ; E)}+\sum_{k=1}^{2}\left(\left\|f_{k}\right\|_{E_{k}}+|\lambda|^{1-\theta_{k}}\left\|f_{k}\right\|_{E}\right)\right] .
\end{aligned}
$$

Proof The uniqueness of a solution of problem (4.2) is obtained from Lemma 4.2. Let us define

$$
\bar{f}(x)= \begin{cases}f(x), & \text { if } x \in[0,1], \\ 0, & \text { if } x \notin[0,1] .\end{cases}
$$

We will show that problem (4.2) has a solution $u \in W^{m+2, p}(0,1 ; E(A), E)$ for $f \in W^{m, p}(0,1$; $E), f_{k} \in E_{k}$ and $u=u_{1}+u_{2}$, where $u_{1}$ is the restriction on $[0,1]$ of the solution of the equation

$$
\left(L_{t}+\lambda\right) u=\bar{f}(x), \quad x \in R=(-\infty, \infty)
$$

and $u_{2}$ is a solution of the problem

$$
\left(L_{t}+\lambda\right) u=0, \quad L_{k t} u=f_{k}-L_{k t} u_{1}
$$

A solution of equation (4.11) is given by

$$
u(x)=F^{-1} L^{-1}(\lambda, t, \xi) F \bar{f}=\frac{1}{2 \pi} \int_{\infty}^{\infty} e^{i \xi x} L^{-1}(\lambda, t, \xi)(F \bar{f})(\xi) d \xi
$$

where $L(\lambda, t, \xi)=A+t \xi^{2}+\lambda$. It follows from the above expression that

$$
\begin{aligned}
& \sum_{j=0}^{m+2} t^{\frac{j}{m+2}}|\lambda|^{1-\frac{j}{m+2}}\left\|u^{(j)}\right\|_{L^{p}(R ; E)}+\|A u\|_{L^{p}(R ; E)} \\
& \quad=\sum_{j=0}^{m+2} t^{\frac{j}{m+2}}|\lambda|^{1-\frac{j}{m+2}}\left\|F^{-1} \xi^{j} L^{-1}(\lambda, t, \xi) F \bar{f}\right\|_{L^{p}(R ; E)}+\left\|F^{-1} A L^{-1}(\lambda, t, \xi) F \bar{f}\right\|_{L^{p}(R ; E)} .
\end{aligned}
$$

It is sufficient to show that the operator-functions

$$
\begin{aligned}
& \Psi_{t \lambda}(\xi)=A L^{-1}(\lambda, t, \xi)\left(1+\xi^{m}\right)^{-1}, \\
& \sigma_{t \lambda}(\xi)=\sum_{j=0}^{m+2} t^{\frac{j}{m+2}}|\lambda|^{1-\frac{j}{m+2}} \xi^{j} L^{-1}(\lambda, t, \xi)\left(1+\xi^{m}\right)^{-1}
\end{aligned}
$$


are uniform Fourier multipliers in $L^{p}(R ; E)$. Actually, due to the positivity of $A$, we have

$$
\begin{aligned}
& \left\|L^{-1}(\lambda, t, i \xi)\right\| \leq M\left(1+t \xi^{2}+|\lambda|\right)^{-1}, \\
& \left\|\Psi_{t, \lambda}(\xi)\right\|=\left\|A\left[A+\lambda+t \xi^{2}\right]^{-1}\right\| \leq C_{1} .
\end{aligned}
$$

It is clear to observe that

$$
\xi \frac{d}{d \xi} \Psi_{t \lambda}(\xi)=-2 t \xi^{2} A L^{-2}(\lambda, t, \xi)=\left[-2 t \xi^{2} L^{-1}(\lambda, t, \xi)\right] A L^{-1}(\lambda, t, \xi) .
$$

Due to $R$-positivity of the operator $A$, the sets

$$
\left\{-2 t \xi^{2}\left[A+t \xi^{2}+\lambda\right]^{-1}\right\}, \quad\left\{A\left[A+t \xi^{2}+\lambda\right]^{-1}\right\}, \quad \xi \in \mathbb{R} \backslash\{0\}
$$

are $R$-bounded. Then, in view of the Kahane contraction principle, from the product properties of the collection of $R$-bounded operators (see, e.g., [36] Lemma 3.5, Proposition 3.4), we obtain

$$
\begin{aligned}
& R\left\{\xi^{i} \frac{d^{i}}{d \xi^{i}} \Psi_{t \lambda}(\xi): \xi \in \mathbb{R} \backslash\{0\}\right\} \leq C_{1}, \\
& R\left\{\xi^{i} \frac{d^{i}}{d \xi^{i}} \sigma_{t \lambda}(\xi): \xi \in \mathbb{R} \backslash\{0\}\right\} \leq C_{2}, \quad i=0,1 .
\end{aligned}
$$

By [33, Theorem 3.4] it follows that $\Psi_{t, \lambda}(\xi)$ and $\sigma_{t \lambda}(\xi)$ are the uniform collection of multipliers in $L^{p}(R ; E)$. Then in view of (4.13) we obtain that problem (4.11) has a solution $u \in W^{m+2, p}(R ; E(A), E)$ and the uniform coercive estimate holds

$$
\sum_{j=0}^{m+2} t^{\frac{j}{m+2}}|\lambda|^{1-\frac{j}{m+2}}\left\|u^{(j)}\right\|_{L^{p(R ; E)}}+\|A u\|_{L^{p}(R ; E)} \leq C\|\bar{f}\|_{L^{p}(R ; E)} .
$$

Let $u_{1}$ be the restriction of $u$ on $(0,1)$. The estimate (4.16) implies that $u_{1} \in W^{m+2, p}(0,1$; $E(A), E)$. By virtue of Lemma 4.1, we get

$$
u_{1}^{\left(m_{k}\right)}(\cdot) \in(E(A) ; E)_{\theta_{k}, p}, \quad k=1,2 .
$$

Hence, $L_{k t} u_{1} \in E_{k}$. Thus, by virtue of Lemma 4.2, problem (4.12) has a unique solution $u_{2}(x)$ that belongs to the space $W^{m+2, p}(0,1 ; E(A), E)$ and

$$
\begin{aligned}
& \sum_{j=0}^{m+2} t^{\frac{j}{m+2}}|\lambda|^{1-\frac{j}{m+2}}\left\|u_{2}^{(j)}\right\|_{L^{p}(0,1 ; E)}+\left\|A u_{2}\right\|_{L^{p}(0,1 ; E)} \\
& \leq C \sum_{k=1}^{2}\left[\left\|f_{k}\right\|_{E_{k}}+|\lambda|^{1-\theta_{k}}\left\|f_{k}\right\|_{E}+t^{\theta_{k}}\left\|u_{1}^{\left(m_{k}\right)}\right\|_{C\left([0,1] ; E_{k}\right)}+t^{\theta_{k}}|\lambda|^{1-\theta_{k}}\left\|u_{1}\right\|_{C([0,1] ; E)}\right] .
\end{aligned}
$$

Moreover, from (4.16) we obtain

$$
\sum_{j=0}^{m+2} t^{\frac{j}{m+2}}|\lambda|^{1-\frac{j}{m+2}}\left\|u_{1}^{(j)}\right\|_{L^{p}(0,1 ; E)}+\left\|A u_{1}\right\|_{L^{p}(0,1 ; E)} \leq C\|f\|_{W^{m, p}(0,1 ; E)} .
$$


Therefore, by Lemma 4.1 and by estimate (4.17), we obtain

$$
t^{\theta_{k}}\left\|u_{1}^{\left(m_{k}\right)}(\cdot)\right\|_{E_{k}} \leq C\left\|u_{1}\right\|_{W_{t}^{m+2, p}(0,1 ; E(A), E)} \leq C\|f\|_{W^{m, p}(0,1 ; E)} .
$$

So, in view of Lemma 4.1 and estimates (4.17)-(4.19), we get

$$
\begin{aligned}
& \sum_{j=0}^{m+2} t^{\frac{j}{m+2}}|\lambda|^{1-\frac{j}{m+2}}\left\|u_{2}^{(j)}\right\|_{L^{p}(0,1 ; E)}+\left\|A u_{2}\right\|_{L^{p}(0,1 ; E)} \\
& \leq C\left(\|f\|_{L^{p}(0,1 ; E)}+\sum_{k=1}^{2}\left(\left\|f_{k}\right\|_{E_{k}}+|\lambda|^{1-\theta_{k}}\left\|f_{k}\right\|_{E}\right)\right) .
\end{aligned}
$$

Finally, from (4.18) and (4.20) we obtain (4.10).

Now, we can prove the main result of this section.

Proof of Theorem 4.1 Let $G_{2}=\left(0, b_{1}\right) \times\left(0, b_{2}\right)$. It is clear to see that

$$
W^{m, q}\left(G_{2} ; E\right)=W^{m, q}\left(0, b_{1} ; X_{0}, X\right)=W^{m, q}\left(0, b_{1} ; X\right) \cap L^{q}\left(0, b_{1} ; X_{0}\right),
$$

where $X_{0}=W^{m, q}\left(0, b_{2} ; E\right)$ and $X=L^{q}\left(0, b_{2} ; E\right)$.

Let us consider the BVP

$$
-\varepsilon_{1} \frac{\partial^{2} u}{\partial x_{1}^{2}}-\varepsilon_{2} \frac{\partial^{2} u}{\partial x_{2}^{2}}+(A+\lambda) u\left(x_{1}, x_{2}\right)=f\left(x_{1}, x_{2}\right), \quad L_{k j \varepsilon} u=0, \quad k, j=1,2,
$$

where $L_{k j \varepsilon}$ are defined by equalities (1.5). Problem (4.21) can be expressed as the following BVP for an ordinary DOE:

$$
L u=-\varepsilon_{1} \frac{d^{2} u}{d x_{1}^{2}}+\left(B_{\varepsilon_{2}}+\lambda\right) u\left(x_{2}\right)=f\left(x_{2}\right), \quad x_{1} \in\left(0, b_{1}\right), \quad L_{k 1 \varepsilon_{1}} u=0
$$

where $L_{k 1 \varepsilon_{1}}$ are boundary conditions of type (3.2), $B_{\varepsilon_{2}}$ is the operator acting in $X_{0}$ and $X$ defined by

$$
\begin{aligned}
& B_{\varepsilon_{2}} u=-\varepsilon_{2} \frac{d^{2} u}{d x_{2}^{2}}+A u\left(x_{2}\right), \quad D\left(B_{\varepsilon_{2}}\right)=W^{m+2, q}\left(0, b_{2} ; E(A), E, L_{2 k \varepsilon_{2}}\right), \\
& W^{2, q}\left(0, b_{2} ; E(A), E, L_{2 k \varepsilon_{2}}\right) .
\end{aligned}
$$

Since $X_{0}$ and $X$ are UMD spaces, (see, e.g., [35, Theorem 4.5.2]) by virtue of Theorem 4.2, we obtain that problem (4.22) has a unique solution $u \in W^{m+2, q}\left(0, b_{1} ; D\left(B_{\varepsilon_{2}}\right), X\right)$ for $f \in$ $W^{m, q}\left(0, b_{1} ; X\right)$ and $\lambda \in S_{\psi, \varkappa}$ with sufficiently large $\varkappa>0$. Moreover, the coercive uniform estimates holds

$$
\begin{aligned}
& \sum_{i=0}^{m+2} \varepsilon_{1}^{\frac{i}{m+2}}|\lambda|^{1-\frac{i}{m+2}}\left\|u^{(i)}\right\|_{L^{q}\left(0, b_{1} ; X\right)}+\left\|B_{\varepsilon_{2}} u\right\|_{L^{q}\left(0, b_{1} ; X\right)} \leq C\|f\|_{W^{m}, q\left(0, b_{1} ; X\right)}, \\
& \sum_{i=0}^{2} \varepsilon_{1}^{\frac{i}{2}}|\lambda|^{1-\frac{i}{2}}\left\|u^{(i)}\right\|_{L^{q}\left(0, b_{1} ; X_{0}\right)}+\left\|B_{\varepsilon_{2}} u\right\|_{L^{q}\left(0, b_{1} ; X_{0}\right)} \leq C\|f\|_{L^{q}\left(0, b_{1} ; X_{0}\right)} .
\end{aligned}
$$


From (4.23) we obtain that problem (4.22) has a unique solution

$$
u \in W^{m+2, q}\left(G_{2} ; E(A), E\right) \text { for } W^{m, q}\left(G_{2} ; E\right) .
$$

Moreover, the uniform coercive estimates hold

$$
\sum_{i=0}^{m+2} \varepsilon_{1}^{\frac{i}{m+2}}|\lambda|^{1-\frac{i}{m+2}}\left\|u^{(i)}\right\|_{L^{q}\left(0, b_{1} ; X\right)}+\left\|B_{\varepsilon_{2}} u\right\|_{L^{q}\left(0, b_{1} ; X\right)} \leq C\|f\|_{W^{m, q}\left(G_{2} ; E\right)} .
$$

By applying Theorem 4.2 for $f_{k}=0$ and $E=X$, we get the following uniform estimate:

$$
\sum_{j=0}^{m+2} \varepsilon_{2}^{\frac{i}{m+2}}|\lambda|^{1-\frac{i}{m+2}}\left\|u^{(i)}\right\|_{X}+\|A u\|_{X} \leq C\left\|B_{\varepsilon_{2}} u\right\|_{W^{m, q}\left(0, b_{2} ; E\right)}
$$

From estimates (4.24)-(4.25) we conclude the corresponding claim for problem (4.21). Then, by continuing this process $n$-times, we obtain the assertion.

\section{Nonlocal initial-boundary value problems for the Stokes system with small parameters}

In this section, we show the uniform maximal regularity properties of the nonlocal initial value problem for nonstationary Stokes equations (1.6).

The function $u \in W_{\sigma}^{2, q}\left(G, L_{k j \varepsilon}\right)=\left\{u \in W^{2, q}\left(G ; R^{n}\right), L_{k j \varepsilon} u=0\right.$, $\left.\operatorname{div} u=0\right\}$ satisfying equation (1.6) a.e. on $G$ is called the stronger solution of problem (1.6).

Let $W^{s, q}(G), 0<s<\infty$ be the Sobolev space of order $s$ such that $W^{0, q}(G)=L^{q}(G)$. For $q \in(1, \infty)$, let $X_{q}=L_{\sigma}^{q}(G)$ denote the closure of $C_{0 \sigma}^{\infty}(G)$ in $L^{p}\left(G ; R^{n}\right)$, where

$$
C_{0 \sigma}^{\infty}(G)=\left\{u \in C_{0}^{\infty}(G), \operatorname{div} u=0\right\} .
$$

It is known that ( see, e.g., Fujiwara and Morimoto [17]) a vector field $u \in L^{q}\left(G ; R^{n}\right)$ has the Helmholtz decomposition, i.e., all $u \in L^{q}\left(G ; R^{n}\right)$ can be uniquely decomposed as $u=$ $u_{0}+\nabla \varphi$ with $u_{0} \in L_{\sigma}^{q}(G), u_{0}=P_{q} u$, where $P_{q}=P$ is a projection operator from $L^{q}\left(G ; R^{n}\right)$ to $L_{\sigma}^{q}(G)$ and $\varphi \in L_{\mathrm{loc}}^{q}(\bar{G}), \nabla \varphi \in L^{q}\left(G ; R^{n}\right)$, so that

$$
\|\nabla \varphi\|_{q} \leq C\|u\|_{q}, \quad\|\varphi\|_{L^{q}(G \cap B)} \leq C\|u\|_{q}
$$

with $C$ independent of $u$, where $B$ is an open ball in $R^{n}$ and $\|u\|_{p}$ denotes the norm of $u$ in $L^{q}\left(G ; R^{n}\right)$ or $L^{q}(G)$.

Then problem (1.6) can be reduced to the following BVP:

$$
\begin{aligned}
& -P \triangle_{\varepsilon} u+\lambda u=f(x), \quad x \in G, \\
& L_{k j \varepsilon} u=\sum_{i=0}^{m_{k j}} \varepsilon_{k}^{\sigma_{i k}}\left[\alpha_{k j i} \frac{\partial^{i} u}{\partial x_{k}^{i}}\left(G_{k 0}\right)+\beta_{k j i} \frac{\partial^{i} u}{\partial x_{k}^{i}}\left(G_{k b}\right)\right]=0, \\
& \quad k=1,2, \ldots, n, j=1,2 .
\end{aligned}
$$


Consider the parameter-dependent Stokes operator $O_{\varepsilon}=O_{\varepsilon, q}$ generated by problem (5.1), i.e.,

$$
D\left(O_{\varepsilon}\right)=\left(W_{\sigma}^{2, q}\left(G ; L_{k j}\right)\right)^{n}, \quad O_{\varepsilon} u=-P \triangle_{\varepsilon} u .
$$

From Corollary 3.1 we get that the operator $O_{\varepsilon}$ is positive and also is a generator of a bounded holomorphic semigroup $S_{\varepsilon}(t)=\exp \left(-O_{\varepsilon} t\right)$ for $t>0$.

In a similar way as in [18], we show the following.

Proposition 5.1 The following estimate holds:

$$
\left\|O_{\varepsilon}^{\alpha} S_{\varepsilon}(t)\right\| \leq C t^{-\alpha}
$$

uniformly in $\varepsilon=\left(\varepsilon_{1}, \varepsilon_{2}, \ldots, \varepsilon_{n}\right)$ for $\alpha \geq 0$ and $t>0$.

Proof From Result 3.1 we obtain that the operator $O_{\varepsilon}$ is uniformly positive in $L_{q}\left(G ; R^{n}\right)$, i.e., for $\lambda \in S_{\psi, \varkappa}, 0<\psi<\pi$, the following estimate holds:

$$
\left\|\left(O_{\varepsilon}+\lambda\right)^{-1}\right\| \leq \frac{M}{|\lambda|}
$$

where the constant $M$ is independent of $\lambda$ and $\varepsilon$. Then, by using the Danford integral and operator calculus as in [18], we obtain the assertion.

Now consider problem (1.7). The main theorem in this section is the following.

Theorem 5.1 Let $0<\varepsilon_{k} \leq 1,(-1)^{m_{k 1}} \alpha_{k 1} \beta_{k 2}-(-1)^{m_{k 2}} \alpha_{k 2} \beta_{k 1} \neq 0$ and $p, q \in(1, \infty)$. Then there is a unique solution $(u, \nabla \varphi)$ of problem $(1.7)$ for $f \in L^{p}\left(0, T ; L^{q}\left(G ; R^{n}\right)\right)=B(p, q)$ and $a \in B_{p, q}^{2-\frac{2}{p}}$. Moreover, the following uniform estimate holds:

$$
\left\|\frac{\partial u}{\partial t}\right\|_{B(p, q)}+\sum_{k=1}^{n}\left\|\varepsilon_{k} \frac{\partial^{2} u}{\partial x_{k}^{2}}\right\|_{B(p, q)}+\|\nabla \varphi\|_{B(p, q)} \leq C\|f\|_{B(p, q)}+\|a\|_{\substack{B_{p, q}^{2-\frac{2}{p}}(G) \\ \text { (a) }}}
$$

with $C=C(T, G, p, q)$ independent off and $\varepsilon$.

Proof Problem (1.7) can be expressed as the following abstract parabolic problem with a small parameter:

$$
\frac{d u}{d t}+O_{\varepsilon} u=f(t), \quad u(0)=a .
$$

If we put $E=L^{q}\left(G ; R^{n}\right)$ and $A=\varkappa>0$ in Theorem 3.1, then the Result 3.1 implies that the operator $O_{\varepsilon}$ is uniformly positive and generates bounded holomorphic semigroup in $L^{q}\left(G ; R^{n}\right)$ uniformly in $\varepsilon_{k}$. Moreover, by using [41, Theorem 3.1 ] we get that operator $O_{\varepsilon}$ is $R$-positive in $E$. Since $E$ is a UMD space, in a similar way as in [33, Theorem 4.2], we obtain that for $f \in L^{p}(0, T ; E)$ and $a \in\left(D\left(O_{\varepsilon}\right), E\right)_{\frac{1}{p}, p}$, there is a unique solution $u \in W^{1, p}\left(0, T, D\left(O_{\varepsilon}\right), E\right)$ of problem (5.3) so that the following uniform estimate holds:

$$
\left\|\frac{d u}{d t}\right\|_{L^{p}(0, T ; E)}+\left\|O_{\varepsilon} u\right\|_{L^{p}(0, T ; E)} \leq C\left(\|f\|_{L^{p}(0, T ; E)}+\|a\|_{\left(D\left(A_{\varepsilon}\right), E\right)_{\bar{p}, p}}\right) .
$$


From (5.4) for all $u \in W_{\sigma}^{2, q}\left(G, L_{k j \varepsilon}\right)$, we get the following estimate:

$$
\sum_{k=1}^{n} \varepsilon_{k}\left\|\frac{\partial^{2} u}{\partial x_{k}^{2}}\right\|_{q} \leq C\left\|A_{\varepsilon} u\right\|_{q}
$$

uniformly in $\varepsilon=\left(\varepsilon_{1}, \varepsilon_{2}, \ldots, \varepsilon_{n}\right)$.

\section{Existence and uniqueness for the Navier-Stokes equation with parameters}

In this section, we study the Navier-Stokes problem (1.1)-(1.3) in the space $X_{q}$. Problem (1.1)-(1.3) can be expressed as

$$
\frac{d u}{d t}+O_{\varepsilon} u=F u+P f, \quad u(0)=0, \quad t>0
$$

where

$$
F u=-P(u, \nabla) u
$$

We consider equation (6.1) in an integral form

$$
u(t)=S_{\varepsilon}(t) a+\int_{0}^{t} S_{\varepsilon}(t-s)[F u(s)+P f(s)] d s, \quad t>0 .
$$

To prove the main result, we need the following result which are obtained in a similar way as in [11, Theorem 2].

Lemma 6.1 For any $0 \leq \alpha \leq 1$, the domain $D\left(O_{\varepsilon}^{\alpha}\right)$ is the complex interpolation space $\left[X_{q}, D\left(O_{\varepsilon}\right)\right]_{\alpha}$.

Lemma 6.2 For each $k=1,2, \ldots, n$, the operator $u \rightarrow O_{\varepsilon}^{-\frac{1}{2}} P\left(\frac{\partial}{\partial x_{k}}\right)$ u extends uniquely to a uniformly bounded linear operator from $L^{q}\left(G ; R^{n}\right)$ to $X_{q}$.

Proof Since $O_{\varepsilon}$ is a positive operator, it has fractional powers $O_{\varepsilon}^{\alpha}$. From Lemma 6.1, it follows that the domain $D\left(O_{\varepsilon}^{\alpha}\right)$ is continuously embedded in $X_{q} \cap H_{q}^{2 \alpha}\left(G ; R^{n}\right)$ for any $\alpha>0$, where $H_{q}^{2 \alpha}\left(G ; R^{n}\right)$ is the vector-valued Bessel space. Then, by using the duality argument and due to uniform positivity of $O_{\varepsilon}^{\frac{1}{2}}$, we obtain the following uniformly in $\varepsilon$ estimate:

$$
\left\|O_{\varepsilon}^{-\frac{1}{2}} P\left(\frac{\partial}{\partial x_{k}}\right) u\right\|_{L^{q\left(G ; R^{n}\right)}} \leq C\|u\|_{X_{q}}
$$

By reasoning as in [12], we obtain the following.

Lemma 6.3 Let $0 \leq \delta<\frac{1}{2}+\frac{n}{2}\left(1-\frac{1}{q}\right)$. Then the following estimate holds:

$$
\left\|\varepsilon O_{\varepsilon}^{-\delta} P(u, \nabla) v\right\|_{q} \leq M\left\|O_{\varepsilon}^{\theta} u\right\|_{q}\left\|O_{\varepsilon}^{\sigma} u\right\|_{q}
$$

uniformly in $\varepsilon$ with some constant $M=M(\delta, \theta, q, \sigma)$ provided that $\theta>0, \sigma>0, \sigma+\delta>\frac{1}{2}$ and

$$
\theta+\sigma+\delta>\frac{n}{2 q}+\frac{1}{2}
$$


Proof Assume that $0<v<\frac{n}{2}\left(1-\frac{1}{q}\right)$. Since $D\left(O_{\varepsilon}^{\alpha}\right)$ is continuously embedded in $X_{q} \cap$ $H_{q}^{2 \alpha}\left(G ; R^{n}\right)$, and since $L^{q^{\prime}}\left(G ; R^{n}\right) \cap X_{q^{\prime}}$ is the same as $X_{s^{\prime}}$, by the Sobolev embedding theorem, we obtain that the operator

$$
O_{\varepsilon, q^{\prime}}^{-v}: X_{q^{\prime}} \rightarrow D\left(O_{\varepsilon, q^{\prime}}^{v}\right) \rightarrow X_{s^{\prime}}
$$

is bounded, where

$$
\frac{1}{s^{\prime}}=\frac{1}{q^{\prime}}-\frac{2 v}{n}, \quad \frac{1}{q}+\frac{1}{q^{\prime}}=1
$$

By the duality argument then, we get that the operator $u \rightarrow O_{\varepsilon, q}^{-v}$ is bounded from $X_{s}$ to $X_{q}$, where

$$
\frac{1}{s}=1-\frac{1}{s^{\prime}}=\frac{1}{q}+\frac{2 v}{n}
$$

Consider first the case $\delta>\frac{1}{2}$. Since $P(u, \nabla) v$ is bilinear in $u, v$, it suffices to prove the estimate on a dense subspace. Therefore, assume that $u$ and $v$ are smooth. Since $\operatorname{div} u=0$, we get

$$
(u, \nabla) v=\sum_{k=1}^{n} \frac{\partial}{\partial x_{k}}\left(u_{k} v\right)
$$

Taking $v=\delta-\frac{1}{2}$, using the uniform boundedness of $O_{\varepsilon, q}^{-v}$ from $X_{s}$ to $X_{q}$ and Lemma 6.2 for all $\varepsilon>0$, we obtain

$$
\left\|\varepsilon O_{\varepsilon}^{-\delta} P(u, \nabla) v\right\|_{q}=\left\|\varepsilon O_{\varepsilon, q}^{\frac{1}{2}-v} \sum_{k=1}^{n} P \frac{\partial}{\partial x_{k}}\left(u_{k} v\right)\right\|_{q} \leq\left\|\left|u\|v \mid\|_{s} .\right.\right.
$$

By assumption we can take $r$ and $\eta$ such that

$$
\frac{1}{r} \geq \frac{1}{q}-\frac{2 \theta}{n}, \quad \frac{1}{\eta} \geq \frac{1}{q}-\frac{2 \sigma}{n}, \quad \frac{1}{r}+\frac{1}{\eta}=\frac{1}{s}, \quad r>1, \eta<\infty .
$$

Since $D\left(O_{\varepsilon, q}^{\alpha}\right)$ is continuously embedded in $X_{q} \cap H_{q}^{2 \alpha}\left(G ; R^{n}\right)$, by the Sobolev embedding, we get

$$
\left\|\left|u\|v \mid\|_{s} \leq\|u\|_{r}\|v\|_{\eta} \leq M\left\|O_{\varepsilon, q}^{\theta} u\right\|_{r}\left\|O_{\varepsilon, q}^{\sigma} v\right\|_{\eta},\right.\right.
$$

i.e., we have the required result for $\delta>\frac{1}{2}$. In particular, we get the following uniform estimate:

$$
\left\|\varepsilon O_{\varepsilon}^{-\frac{1}{2}} P(u, \nabla) v\right\|_{q} \leq M\left\|O_{\varepsilon, q}^{\theta} u\right\|_{r}\left\|O_{\varepsilon, q}^{\sigma} v\right\|_{\eta}, \quad \theta+\beta \geq \frac{n}{2 q}, \quad \beta>0
$$

Similarly, we obtain

$$
\|\varepsilon P(u, \nabla) v\|_{q} \leq C\|u\|_{r}\|v\|_{\eta} \leq C\left\|O_{\varepsilon, q}^{\theta} u\right\|_{r}\left\|O_{\varepsilon, q}^{\beta+\frac{1}{2}} v\right\|_{\eta}
$$


for $\frac{1}{r}+\frac{1}{\eta}=\frac{1}{q}$ and $\delta=0$. The above two estimates show that the map $v \rightarrow P(u, \nabla) v$ is a uniform bounded operator from $D\left(O_{\varepsilon}^{\beta}\right)$ to $D\left(O_{\varepsilon}^{-\frac{1}{2}}\right)$ and from $D\left(O_{\varepsilon}^{\beta+\frac{1}{2}}\right)$ to $X_{q}$. By using Lemma 6.1 and the interpolation of Banach spaces $[30, \S 1.3 .2]$ for $0 \leq \delta \leq \frac{1}{2}$, we obtain

$$
\|\varepsilon P(u, \nabla) v\|_{q} \leq C\left\|O_{\varepsilon, q}^{\theta} u\right\|_{r}\left\|O_{\varepsilon, q}^{\sigma} v\right\|_{\eta} .
$$

By using Lemma 6.3 and the iteration argument, by reasoning as in Fujita and Kato [18], we obtain the following.

Theorem 6.1 Let $0<\varepsilon_{k} \leq 1,(-1)^{m_{k 1}} \alpha_{k 1} \beta_{k 2}-(-1)^{m_{k 2}} \alpha_{k 2} \beta_{k 1} \neq 0$. Let $\gamma<1$ be a real number and $\delta \geq 0$ such that

$$
\frac{n}{2 q}-\frac{1}{2} \leq \gamma, \quad-\gamma<\delta<1-|\gamma|
$$

Suppose that $a \in D\left(O_{\varepsilon}^{\gamma}\right)$, and that $\left\|O_{\varepsilon}^{-\delta} P f(t)\right\|$ is continuous on $(0, T)$ and satisfies

$$
\left\|O_{\varepsilon}^{-\delta} P f(t)\right\|=o\left(t^{\gamma+\delta-1}\right) \quad \text { as } t \rightarrow 0 .
$$

Then there is $T_{*} \in(0, T)$ independent of $\varepsilon$ and a local solution of (6.2) such that

(1) $u \in C\left(\left[0, T_{*}\right] ; D\left(O_{\varepsilon}^{\gamma}\right)\right), u(0)=a$;

(2) $u \in C\left(\left(0 T_{*}\right] ; D\left(O_{\varepsilon}^{\alpha}\right)\right)$ for some $T_{*}>0$;

(3) $\left\|O_{\varepsilon}^{\alpha} u(t)\right\|=o\left(t^{\gamma-\alpha}\right)$ as $t \rightarrow 0$ for all $\alpha$ with $\gamma<\alpha<1-\delta$ uniformly with respect to the parameter $\varepsilon$.

Moreover, the solution of (5.2) is unique if

(4) $u \in C\left(\left(0 T_{*}\right] ; D\left(O_{\varepsilon}^{\beta}\right)\right)$;

(5) $\left\|O_{\varepsilon}^{\alpha} u(t)\right\|=o\left(t^{\gamma-\beta}\right)$ as $t \rightarrow 0$ for some $\beta$ with $\beta>|\gamma|$ uniformly in $\varepsilon$.

Proof We introduce the following iteration scheme:

$$
\begin{aligned}
& u_{0}(t)=S_{\varepsilon}(t) a+\int_{0}^{t} S_{\varepsilon}(t-s) P f(s) d s, \\
& u_{m+1}(t)=u_{0}(t)+\int_{0}^{t} S_{\varepsilon}(t-s) F u_{m}(s) d s, \quad m \geq 0 .
\end{aligned}
$$

By estimating the term $u_{0}(t)$ in (6.4) and by using Proposition 5.1 for $\gamma \leq \alpha<1-\delta$, we get

$$
\begin{aligned}
\left\|O_{\varepsilon}^{\alpha} u_{0}(t)\right\| & \leq\left\|O_{\varepsilon}^{\alpha} S_{\varepsilon}(t) a\right\|+\int_{0}^{t}\left\|O_{\varepsilon}^{\alpha+\delta} S_{\varepsilon}(t-s)\right\|\left\|O_{\varepsilon}^{-\delta} P f(s)\right\| d s \\
& \leq\left\|O_{\varepsilon}^{\alpha} S_{\varepsilon}(t) a\right\|+C_{\alpha+\delta} \int_{0}^{t}\|(t-s)\|^{-(\alpha+\delta)}\left\|O_{\varepsilon}^{-\delta} P f(s)\right\| d s \leq M_{\alpha} t^{\gamma-\alpha}
\end{aligned}
$$

uniformly in $\varepsilon$ with

$$
M_{\alpha}=\sup _{0<t \leq T, \varepsilon>0} t^{\alpha-\gamma}\left\|O_{\varepsilon}^{\alpha+\delta} S_{\varepsilon}(t) a\right\|+C_{\alpha+\delta} N B(1-\delta-\alpha, \gamma+\alpha)
$$


where $N=\sup _{0<t \leq T} t^{1-\gamma-\delta}\left\|O_{\varepsilon}^{-\delta} P f(t)\right\|$ and $B(a, b)$ is the beta function. Here we suppose $\gamma+\delta>0$. By induction assume that $u_{m}(t)$ satisfies the following estimate:

$$
\left\|O_{\varepsilon}^{\alpha} u_{m}(t)\right\| \leq M_{\alpha m} t^{\gamma-\alpha}, \quad \gamma \leq \alpha<1-\delta
$$

We will estimate $O_{\varepsilon}^{\alpha} u_{m+1}(t)$ by using (6.2). To estimate the term $\left\|O_{\varepsilon}^{-\delta} F u_{m}(s)\right\|$, we suppose

$$
\theta+\sigma+\delta=1+\gamma, \quad \gamma<\theta<1-\delta, \gamma<\sigma<1-\delta, \theta>0, \sigma>0, \delta+\sigma>\frac{1}{2}
$$

so that the numbers $\theta, \sigma, \delta$ satisfy the assumptions of Lemma 6.3. Using Lemma 6.3 and (6.5), we get the following uniform estimate:

$$
\left\|O_{\varepsilon}^{-\delta} F u_{m}(s)\right\| \leq C M_{\theta m} M_{\sigma m} s^{\gamma+\delta-1}
$$

Therefore, we obtain

$$
\begin{aligned}
\left\|O_{\varepsilon}^{\alpha} u_{m}(t)\right\| & \leq M_{\alpha} t^{\gamma-\alpha}+M_{\alpha+\delta} \int_{0}^{t}\|(t-s)\|^{-(\alpha+\delta)}\left\|O_{\varepsilon}^{-\delta} F u_{m}(s)\right\| d s \\
& \leq M_{\alpha m+1} t^{\gamma-\alpha}
\end{aligned}
$$

with

$$
M_{\alpha m+1}=M_{\alpha}+M_{\alpha+\delta} M B(1-\delta-\alpha, \gamma+\delta) M_{\theta m} M_{\sigma m}
$$

Since we get the uniform estimates with respect to the parameter $\varepsilon$, the remaining part of the proof is the same as in [12, Theorem 2.3], so this part is omitted.

\section{Competing interests}

The author declares that they have no competing interests.

\section{Acknowledgements}

Dedicated to International Conference on the Theory, Methods and Applications of Nonlinear Equations in Kingsville,

TX-USA Texas A\&M University-Kingsville-2012.

Received: 5 March 2013 Accepted: 12 April 2013 Published: 26 April 2013

\section{References}

1. Beirâo da Veiga, $\mathrm{H}$ : Vorticity and regularity for flows under the Navier boundary condition. Commun. Pure Appl. Anal. 5(4), 907-918 (2006)

2. Hamouda, M, Temam, R: Some Singular Perturbation Problems Related to the Navier-Stokes Equations, Advances in Deterministic and Stochastic Analysis, pp. 197-227. World Scientific, Hackensack (2007)

3. Iftimie, D, Planas, G: Inviscid limits for the Navier-Stokes equations with Navier friction boundary conditions. Nonlinearity 19(4), 899-918 (2006)

4. Lions, J-L: Mathematical Topics in Fluid Mechanics, vol. 1. The Clarendon Press Oxford University Press, New York (1996) Incompressible models, Oxford Science Publications

5. Temam, R, Wang, X: Boundary layers associated with incompressible Navier-Stokes equations: the noncharacteristic boundary case. J. Differ. Equ. 179(2), 647-686 (2002)

6. Xiao, Y, Xin, Z: On the vanishing viscosity limit for the 3D Navier-Stokes equations with a slip boundary condition. Commun. Pure Appl. Math. 60(7), 1027-1055 (2007)

7. Amann, H: On the strong solvability of the Navier-Stokes equations. J. Math. Fluid Mech. 2, 16-98 (2000)

8. Caffarelli, L, Kohn, R, Nirenberg, L: Partial regularity of suitable weak solutions of the Navier-Stokes equations. Commun. Pure Appl. Math. 35, 771-831 (1982)

9. Cannone, M: A generalization of a theorem by Kato on Navier-Stokes equations. Rev. Mat. Iberoam. 13(3), 515-541 (1997)

10. Desch, W, Hieber, M, Prüss, J: $L_{p}$-theory of the Stokes equation in a half-space. J. Evol. Equ. 2001, 1 (2001) 
11. Giga, Y: Domains of fractional powers of the Stokes operator in $L_{r}$ spaces. Arch. Ration. Mech. Anal. 89, 251-265 (1985)

12. Giga, $Y$, Miyakava, T: Solutions in $L_{r}$ of the Navier-Stokes initial value problem. Arch. Ration. Mech. Anal. 89, 267-281 (1985)

13. Giga, Y, Sohr, H: Abstract $L_{p}$ estimates for the Cauchy problem with applications to the Navier-Stokes equations in exterior domains. J. Funct. Anal. 102, 72-94 (1991)

14. Giga, Y: Solutions for semilinear parabolic equation $L^{p}$ and regularity of weak solutions of the Navier-Stokes systems. J. Differ. Equ. 61, 186-212 (1986)

15. Galdi, GP: An Introduction to the Mathematical Theory of the Navier-Stokes Equations I: Linearized Steady Problems, 2nd edn. Springer, Berlin (1998)

16. Fefferman, C, Constantin, P: Direction of vorticity and the problem of global regularity for the 3-d Navier-Stokes equations. Indiana Univ. Math. J. 42, 775-789 (1993)

17. Fujiwara, D, Morimoto, H: An $L_{r}$-theorem of the Helmholtz decomposition of vector fields. J. Fac. Sci., Univ. Tokyo, Sect. 1A, Math. 24, 685-700 (1977)

18. Fujita, H, Kato, T: On the Navier-Stokes initial value problem I. Arch. Ration. Mech. Anal. 16, 269-315 (1964)

19. Fabes, EB, Lewas, JE, Riviere, NM: Boundary value problems for the Navier-Stokes equations. Am. J. Math. 99, 626-668 (1977)

20. Hopf, E: Uber die Anfangswertaufgabe fur die hydrodynamischen Grundgleichungen. Math. Nachr. 4, 213-231 (1950-51)

21. Heywood, JG: The Navier-Stokes equations: on the existence, regularity and decay of solutions. Indiana Univ. Math. J. 29, 639-681 (1980)

22. Kato, T, Fujita, H: On the nonstationary Navier-Stokes system. Rend. Semin. Mat. Univ. Padova 32, 243-260 (1962)

23. Masmoudi, N: Examples of singular limits in hydrodynamics. In: Evolutionary Equations, vol. III. Handb. Differ. Equ., pp. 195-276. Elsevier, Amsterdam (2007)

24. Leray, J: Sur le mouvement d'un liquide visqueux emplissant l'espace. Acta Math. 63, 193-248 (1934)

25. Ladyzhenskaya, OA: The Mathematical Theory of Viscous Incompressible Flow. Gordon and Breach, New York (1969)

26. Solonnikov, V: Estimates for solutions of nonstationary Navier-Stokes equations. J. Sov. Math. 8, 467-529 (1977)

27. Sobolevskii, PE: Study of Navier-Stokes equations by the methods of the theory of parabolic equations in Banach spaces. Sov. Math. Dokl. 5, 720-723 (1964)

28. Shakhmurov, VB: Separable anisotropic differential operators and applications. J. Math. Anal. Appl. 327(2), $1182-1201$ (2006)

29. Teman, R: Navier-Stokes Equations. North-Holland, Amsterdam (1984)

30. Triebel, H: Interpolation Theory. Function Spaces. Differential Operators. North-Holland, Amsterdam (1978)

31. Wiegner, M: Navier-Stokes equations a neverending challenge. Jahresber. Dtsch. Math.-Ver. 101, 1-25 (1999)

32. Weissler, FB: The Navier-Stokes initial value problem in $L_{p}$. Arch. Ration. Mech. Anal. 74, $219-230$ (1980)

33. Weis, L: Operator-valued Fourier multiplier theorems and maximal $L_{p}$ regularity. Math. Ann. 319, 735-758 (2001)

34. Kato, $\mathrm{T}$ : Strong $L_{p}$-solutions of the Navier-Stokes equation in $R^{m}$, with applications to weak solutions. Math. Z. 187, 471-480 (1984)

35. Amann, H: Linear and Quasi-Linear Equations, 1. Birkhäuser, Basel (1995)

36. Denk, R, Hieber, M, Pruss, J: R-boundedness, Fourier multipliers and problems of elliptic and parabolic type. Mem. Am. Math. Soc. 166, 788 (2005)

37. Dore, C, Yakubov, S: Semigroup estimates and non coercive boundary value problems. Semigroup Forum 60, 93-121 (2000)

38. Lunardi, A: Analytic Semigroups and Optimal Regularity in Parabolic Problems. Birkhäuser, Basel (2003)

39. Shakhmurov, VB: Nonlinear abstract boundary value problems in vector-valued function spaces and applications Nonlinear Anal., Theory Methods Appl. 67(3), 745-762 (2006)

40. Shakhmurov, VB: Linear and nonlinear abstract equations with parameters. Nonlinear Anal., Theory Methods Appl. 73, 2383-2397 (2010)

41. Shakhmurov, VB, Shahmurova, A: Nonlinear abstract boundary value problems atmospheric dispersion of pollutants. Nonlinear Anal., Real World Appl. 11(2), 932-951 (2010)

42. Shakhmurov, VB: Coercive boundary value problems for regular degenerate differential-operator equations. J. Math. Anal. Appl. 292(2), 605-620 (2004)

43. Yakubov, S, Yakubov, Ya: Differential-Operator Equations. Ordinary and Partial Differential Equations. Chapman and Hall/CRC, Boca Raton (2000)

doi:10.1186/1687-2770-2013-107

Cite this article as: Shakhmurov: Nonlocal Navier-Stokes problem with a small parameter. Boundary Value Problems 2013 2013:107 